\title{
Preparation and Characterization of Starch Nanoparticles for Controlled Release of Curcumin
}

\author{
Suk Fun Chin, Siti Nur Akmar Mohd Yazid, and Suh Cem Pang \\ Department of Chemistry, Faculty of Resource Science and Technology, Universiti Malaysia Sarawak, 94300 Kota Samarahan, \\ Sarawak, Malaysia
}

Correspondence should be addressed to Suk Fun Chin; sukfunchin@gmail.com

Received 21 June 2013; Accepted 28 October 2013; Published 29 January 2014

Academic Editor: Saad Khan

Copyright (C) 2014 Suk Fun Chin et al. This is an open access article distributed under the Creative Commons Attribution License, which permits unrestricted use, distribution, and reproduction in any medium, provided the original work is properly cited.

\begin{abstract}
Curcumin was loaded onto starch nanoparticles by using in situ nanoprecipitation method and water-in-oil microemulsion system. Curcumin loaded starch nanoparticles exhibited enhanced solubility in aqueous solution as compared to free curcumin. Effects of formulation parameters such as types of reaction medium, types of surfactant, surfactant concentrations, oil/ethanol ratios, loading time, and initial curcumin concentration were found to affect the particle size and loading efficiency (LF) of the curcumin loaded starch nanoparticles. Under optimum conditions, curcumin loaded starch nanoparticles with mean particles size of $87 \mathrm{~nm}$ and maximum loading efficiency of $78 \%$ were achieved. Curcumin was observed to release out from starch nanoparticles in a sustained way under physiological $\mathrm{pH}$ over a period of 10 days.
\end{abstract}

\section{Introduction}

Curcumin is a natural polyphenol found in the rhizomes of turmeric (Curcuma longa) and exhibited antioxidant, antiinflammatory, antiproliferative, anti-invasive, and antiangiogenic activities $[1,2]$. More recently, curcumin has shown to have antitumor effects in many cancer cell lines $[3,4]$ and the clinical effects of curcumin are being studied in human clinical trials and animal models on various conditions and numerous myeloma [5-7]. Despite these promising biomedical properties, free curcumin molecules suffered from low water solubility, which in turn have resulted in poor bioavailability and clinical efficacy [8]. Hence, researchers have attempted to enhance water solubility and bioavailability of curcumin by loading of curcumin in biodegradable polymeric nanoparticles. For instance, curcumin loaded poly(lactic-coglycolic acid) PLGA nanospheres were formulated for prostate cancer therapy [9].

Starch is a well-known, versatile, and inexpensive polysaccharide which has received great attention in drug delivery applications as they are hydrophilic, biodegradable, and biocompatible with tissue and cells [10-14]. Starch nanoparticles with particle sizes in the range of $10-1000 \mathrm{~nm}$ have been extensively studied as controlled release nanocarriers. For instance, propyl starch nanoparticles loaded with different types of drug (flufenamic acid, testosterone, and caffeine) showed enhanced effectiveness upon permeation studies on human skin [15]. Dialdehyde starch nanoparticles (DASNPs) conjugated with 5-fluorouracil (5-Fu) were found to have enhanced breast cancer cell (MF-7) inhibition in vitro as compared to free 5-Fu [16].

Previously, our research groups have successfully prepared starch nanoparticles by a nanoprecipitation method [17]. In this present work, we report loading of curcumin in situ onto native sago starch to form curcumin loaded starch nanoparticles by precipitation in water-in-oil microemulsion. Water-in-oil microemulsion technique offers many advantages as it requires no extreme pressure and no special or expensive equipment [18]. Besides, preparation of nanoparticles in water-in-oil microemulsion system affords nanoparticles with monodispersed particle size distribution $[19,20]$. The potential application of these native starch nanoparticles as controlled release agent for curcumin was studied. Formulation parameters that affected the loading efficiency and release profiles of curcumin were also investigated. 


\section{Experimental}

2.1. Materials. All chemicals were of reagent grade and were used without further purification. Ultrapure water $\left(\sim 18.2 \mathrm{M} \Omega, 25^{\circ} \mathrm{C}\right)$ was obtained from a Water Purifying System (ELGA, Model Ultra Genetic); cyclohexane and absolute ethanol were obtained from HmbG Chemicals (Germany); oleic acid was obtained from Ajax Chemicals (Australia) while curcumin powder, polysorbate 80 (Tween 80) surfactant, and sorbitan monostearate (Span 60) surfactant were obtained from Merck (Germany). Native sago starch powder and sunflower oil were obtained from a local grocery store.

2.2. Loading of Curcumin onto Starch Nanoparticles. The solvent phases essentially consist of absolute ethanol as an organic solvent, oil (oleic acid, sunflower oil, and cyclohexane) as the continuous phase, and surfactant (Tween 80 or Span 60) as an emulsifying agent. About $2.5 \times 10^{-4} \mathrm{M}$ of curcumin was dissolved in $15 \mathrm{~mL}$ of ethanol solution containing of $0.8 \times 10^{-3} \mathrm{M}$ of surfactant, followed by the addition of $5 \mathrm{~mL}$ of oil and the mixture was stirred continuously for $1 \mathrm{~h}$. After $1 \mathrm{~h}, 1 \mathrm{~mL}$ of starch solution ( $1 \% \mathrm{w} / \mathrm{v})$ was added drop-wise to the mixture and stirred for an addition of $1 \mathrm{~h}$. Curcumin was loaded in situ onto starch nanoparticles as the starch nanoparticles formed during the precipitation process. The curcumin loaded starch nanoparticles were collected by centrifugation and the samples were washed several times with ethanol to remove any excess of curcumin that adhered at the surface of the starch nanoparticles. For loading of curcumin onto starch nanoparticles in ethanolic solution without microemulsion system, the same synthesis conditions were employed, except that the loading was carried out in the ethanol solution only.

2.3. Characterization of Curcumin Loaded Starch Nanoparticles. TEM images were obtained using a Transmission Electron Microscope (TEM) (JEOL-2000EX). Starch nanoparticles were deposited onto a formvar-coated copper grid and dried in a desiccator at room temperature. SEM images were recorded using a scanning electron microscope (SEM) (JEOL-SM 6390 LA) operating between 10-15kV with a filament current of about $60-70 \mu \mathrm{A}$. The samples were dropped on stainless steel plates, dried at room temperature, and coated with a layer of platinum using JEOL/JFC-1600 Auto Fine Coater. The UV absorbances of the samples were measured using a UV/Vis spectrophotometer (Jasco V-630). The fluorescence image of curcumin loaded starch nanoparticles was obtained using an Olympus FV300 confocal laser scanning microscope.

2.4. Loading Efficiency of Curcumin. The curcumin loaded starch nanoparticles were separated from the reaction medium by centrifugation and the UV absorbance of free curcumin was measured at a wavelength of $422 \mathrm{~nm}$. The concentration of curcumin was calculated with reference to a regression equation (linear plot with slope of 0.023 ) obtained from constructed calibration curve of curcumin in absolute ethanol solution. The percentage of loading efficiency of curcumin onto starch nanoparticles was calculated based on the following equation [21, 22]:

$$
\begin{aligned}
& \text { Loading efficiency }(\%) \\
& \qquad=\frac{[\text { curcumin }]_{\text {tot }}-[\text { curcumin }]_{\text {free }}}{[\text { curcumin }]_{\text {tot }}} \times 100 .
\end{aligned}
$$

Loading capacity of curcumin onto starch nanoparticles was calculated based on the following equation:

$$
\begin{aligned}
& \text { Loading capacity }(\mathrm{mg} / \mathrm{mg}) \\
& =\frac{[\text { curcumin }]_{\text {tot }}-[\text { curcumin }]_{\text {free }}}{\text { total weight of nanoparticles }} \times 100,
\end{aligned}
$$

where [curcumin] $_{\text {tot }}$ is the concentration of curcumin added and [curcumin $]_{\text {free }}$ is the concentration of curcumin present in the supernatant after centrifugation.

2.5. Curcumin Release Studies. About $50 \mathrm{mg}$ of curcumin loaded starch nanoparticles was placed in $15 \mathrm{~mL}$ of buffer solution at $\mathrm{pH} 7.4$ at $37 \pm 0.5^{\circ} \mathrm{C}[23]$. At predetermined time intervals, the solution was centrifuged, and the supernatant was removed and replaced with the same volume of buffer solution. The amount of curcumin released in the supernatant was determined using a UV/Vis spectrophotometer. The concentration of curcumin released was calculated with reference to the regression equation generated from constructed calibration curve of curcumin in PBS (linear plot with slope of 0.006). The percentage of curcumin released at a specific time was determined based on the following equation $[24,25]$ :

$$
\text { Release of curcumin }(\%)=\frac{[\text { curcumin }]_{\text {rel }}}{[\text { curcumin }]_{\text {load }}} \times 100 \text {, }
$$

where [curcumin $_{\text {rel }}$ is the concentration of curcumin released and [curcumin $]_{\text {load }}$ is the concentration of curcumin loaded into the starch nanoparticles.

2.6. Swelling Studies. The swelling behaviour of the curcumin loaded starch nanoparticle was studied by immersed preweighted dried curcumin loaded starch nanoparticles in $10 \mathrm{~mL}$ of buffer solution ( $\mathrm{pH} \mathrm{7.4)}$ ) at $37 \pm 0.5^{\circ} \mathrm{C}$. The weights of the swollen starch nanoparticles were determined at various time intervals. The swelling ratio was calculated based on the following equation [26]:

$$
\text { Swelling ratio }(\mathrm{SR})=\frac{\left(W_{w}-W_{d}\right)}{W_{d}},
$$

where $W_{w}$ and $W_{d}$ are the wet and dry weight of the starch nanoparticles.

\section{Results and Discussion}

3.1. Loading of Curcumin into Starch Nanoparticles. Figure 1(a) shows that the curcumin powder was insoluble in aqueous media and observed floating on the aqueous media. In contrast, Figure 1(b) shows that curcumin loaded starch 


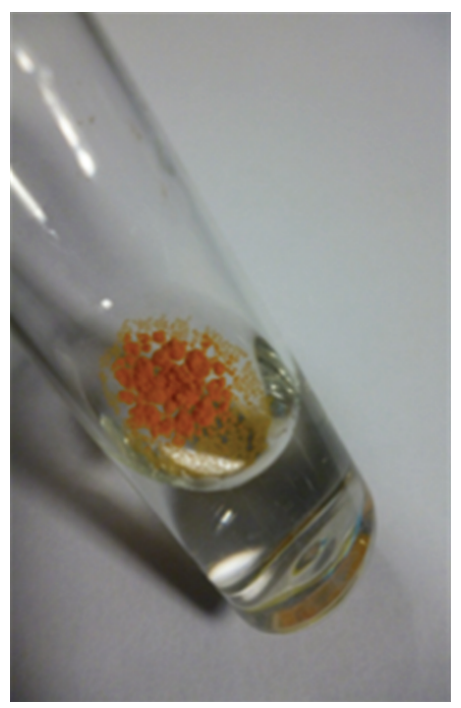

(a)

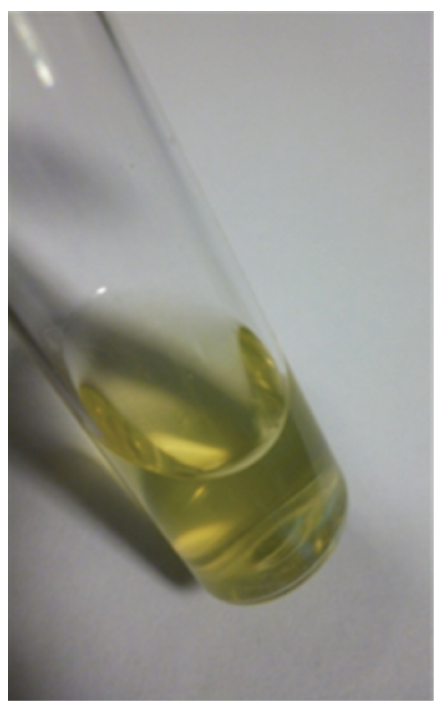

(b)

FIGURE 1: Photographic images of (a) free curcumin which were poorly soluble in aqueous media and macroscopic flakes can be seen floating in the bottle, and (b) curcumin loaded starch nanoparticles were fully dispersible in aqueous media.

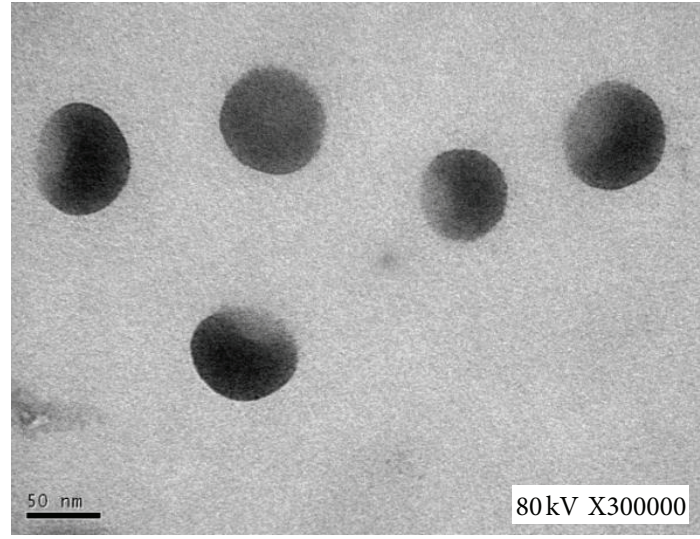

FIGURE 2: TEM image of curcumin loaded starch nanoparticles.

nanoparticles were completely dispersed in aqueous media and rendered curcumin in good dispersibility in aqueous media. The loading of curcumin onto the starch nanoparticles has increased the solubility of curcumin in the aqueous media as the starch nanoparticles were very hydrophilic in nature with small in size and large surface area. It allows for a greater interaction with the aqueous solvent and thus resulted in the increase of curcumin solubility in aqueous media [27, 28].

Figure 2 shows TEM micrograph of curcumin-loaded starch nanoparticles. Curcumin loaded starch nanoparticles were observed to be spherical in shape with mean particle sizes within the range of 50-80 $\mathrm{nm}$.

Figure 3(a) shows the confocal microscopy image of curcumin-loaded starch nanoparticles. The curcuminloaded starch nanoparticles were observed to be fluorescent which confirmed that curcumin had been loaded onto starch nanoparticles. Curcumin was naturally fluorescent in the visible green spectrum whereas starch nanoparticles were transparent [29]. The loading of curcumin onto starch nanoparticles occurred in situ while the starch nanoparticles were precipitated at the interface of reaction medium with curcumin being encapsulated or adsorbed at the same time [30]. In order to further confirm the loading of curcumin onto starch nanoparticles, the UV absorbance of curcumin loaded starch nanoparticles and curcumin solution was measured.

As shown in Figure 3(b), an aqueous solution of curcumin loaded starch nanoparticles showed an absorbance peak at $425.5 \mathrm{~nm}$ (Figure 3(ii)) which was observed to be very close to the characteristic absorbance peak of curcumin at $422 \mathrm{~nm}$ (Figure 3(i)). The red shift in the UV absorbance peak of curcumin loaded starch nanoparticles was probably attributed to the formation of intermolecular hydrogen bondings between curcumin and starch nanoparticles [31, 32].

\subsection{Optimization of Loading Efficiency of Curcumin}

3.2.1. Effect of Reaction Medium. The effects of reaction medium on the loading efficiency of curcumin onto starch nanoparticles were studied by conducting out the loading process in four different reaction media, namely, ethanolic solution, cyclohexane/ethanol microemulsion solution, sunflower oil/ethanol microemulsion solution, and oleic acid/ ethanol microemulsion solution. Figure 4 shows that curcumin loading efficiency of $78 \%$ and $48 \%$ was achieved in oleic acid/ethanol microemulsion and sunflower oil/ethanol microemulsion, respectively. However, low loading efficiency was obtained in the cyclohexane/ethanol microemulsion (17\%) and ethanolic solution (13\%) reaction media.

The microemulsion reaction media have been shown to enhance the loading efficiency of curcumin onto starch nanoparticles due to the presence of lipophilic domains of the 


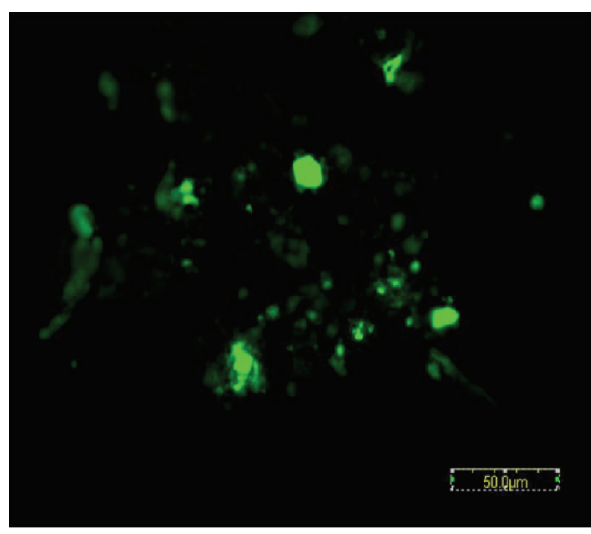

(a)

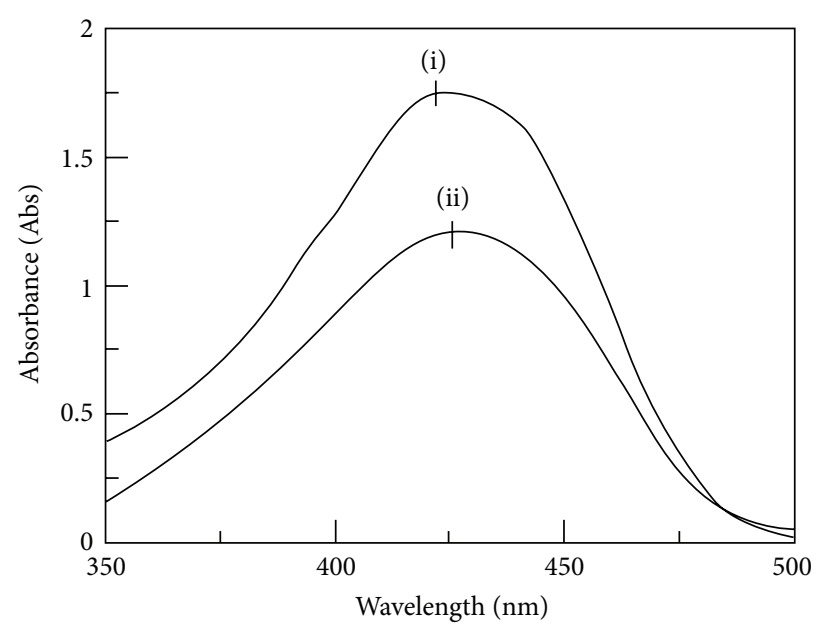

(b)

FIgURE 3: (a) Fluorescent image of curcumin loaded starch nanoparticles and (b) UV spectra of (i) curcumin solution and (ii) curcumin loaded starch nanoparticles.

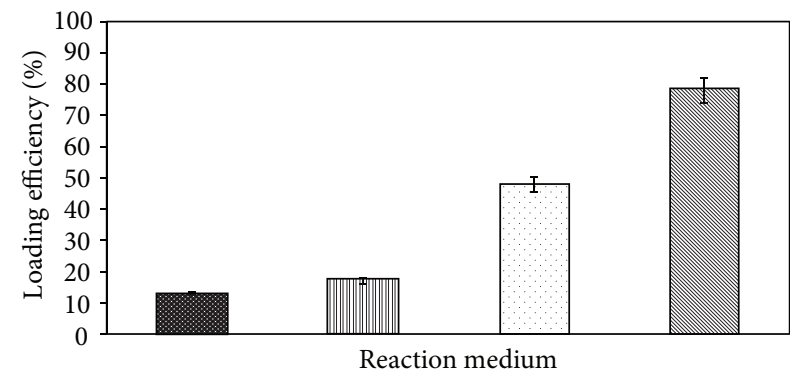

Ethanolic solution

皿 Cyclohexane/ethanol microemulsion

$\square$ Sunflower oil/ethanol microemulsion

$\square$ Oleic acid/ethanol microemulsion

FIGURE 4: Effect of reaction medium on loading efficiency of curcumin onto starch nanoparticles.

microemulsion system which could dissolve the hydrophobic curcumin molecules [33,34]. Lipophilic domains (hydrophobic) were formed due to the presence of oil phase (cyclohexane, sunflower oil, and oleic acid) in the system. Preparation of curcumin loaded starch nanoparticles in oleic acid/ethanol microemulsion reaction medium gave rise to the highest loading efficiency as the highly hydrophobic nature of oleic acid $\left(\mathrm{C}_{18}\right)$ would enable curcumin molecules to be more soluble as compared to in sunflower oil and cyclohexane reaction medium.

Besides, the smallest mean particle sizes of curcuminloaded starch nanoparticles were obtained when oleic acid/ethanol microemulsion was used as the reaction medium (Table 1). This was due to the deep penetration of oleic acid into the surfactant with its longest alkyl chain length and smallest lipophilic domains and thus resulted in the formation of the smallest mean particle sizes of starch nanoparticles by controlling the droplet size [35].
TABLE 1: Effects of reaction medium on mean particles diameter of curcumin loaded starch nanoparticles.

\begin{tabular}{lc}
\hline Reaction medium & Mean particles diameter $(\mathrm{nm})$ \\
\hline Ethanolic & 171 \\
Cyclohexane/ethanol & 137 \\
Sunflower oil/ethanol & 91 \\
Oleic acid/ethanol & 83 \\
\hline
\end{tabular}

3.2.2. Effect of Types of Surfactant. The effect of types of surfactant on the loading efficiency of curcumin onto starch nanoparticles were studied by using two types of nonionic surfactant, namely, sorbitan monostearate (Span 60) and polysorbate 80 (Tween 80 ). The concentrations of both Span 60 and Tween 80 were $0.8 \times 10^{-3} \mathrm{M}$. Nonionic surfactants were used in this study because they are not ionic strength dependence and they are nontoxic in nature [36, 37]. Tween 80 was shown to be the more effective surfactant as compared to Span 60 for loading of curcumin onto starch nanoparticles as evidenced by the higher loading efficiency (78\%) obtained when Tween 80 was used (Figure 5).

The higher loading efficiency could be due to a more stable microemulsion formed in the presence of Tween 80 as it was more soluble in water as compared to Span $60[38,39]$. The hydrophilic polyesters groups of Tween 80 rendered them water soluble, whereas Span 60 was comparatively more hydrophobic and only soluble in hot water. Tween 80 surfactant formed strong hydrogen bonds between its oxyethylene groups and hydroxyl groups of water molecules resulting in a stable water-in-oil microemulsion system and enhanced curcumin loading efficiency. Besides, the longer hydrophobic chain length of Tween $80\left(\mathrm{C}_{64}\right)$ as compared to Span 60 surfactant $\left(\mathrm{C}_{24}\right)$ resulted in deeper penetration of the alkyl chain of the Tween 80 in the oleic acid oil (hydrophobic domains), which promoted the formation of 


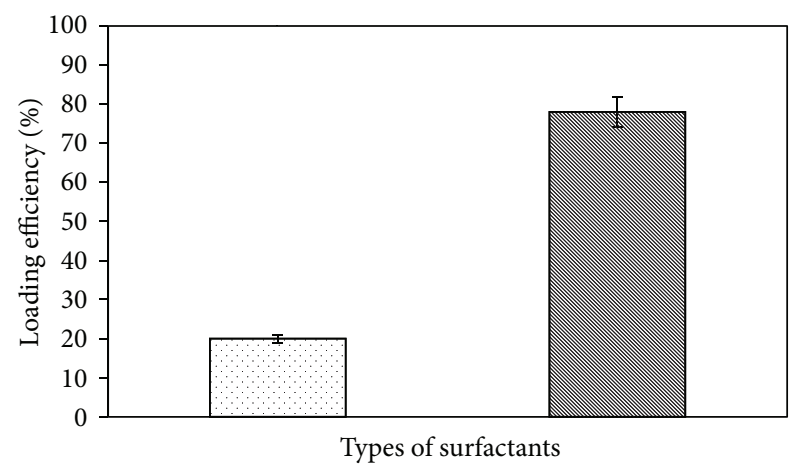

Span 60

$\square$ Tween 80

Figure 5: Effect of types of surfactant on loading efficiency of curcumin onto starch nanoparticles.

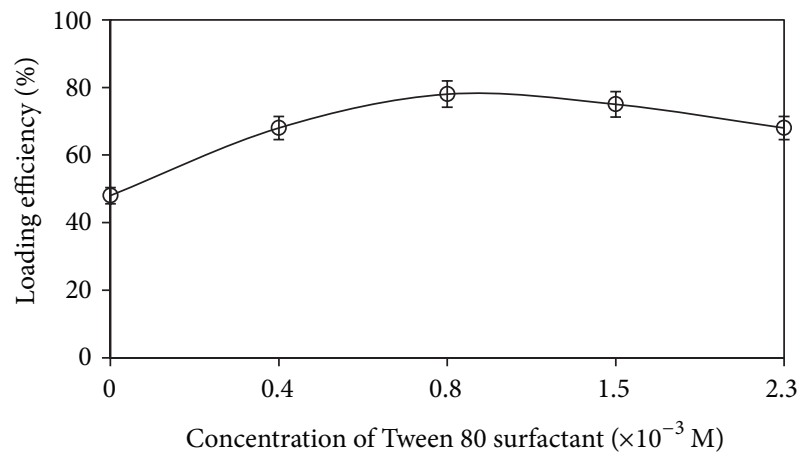

FIGURE 6: Effect of surfactant concentration on loading efficiency of curcumin onto starch nanoparticles.

a stable microemulsion system and enhanced the loading efficiency of curcumin onto starch nanoparticles.

3.2.3. Effect of Surfactant Concentration. The concentration of surfactant in microemulsion system was observed to affect the loading efficiency of curcumin onto starch nanoparticles. As could be seen in Figure 6, the highest loading efficiency of curcumin $(78 \%)$ was achieved when Tween 80 was used as a surfactant in the microemulsion system, whereas the lowest loading efficiency of curcumin (48\%) was obtained when no any surfactant was used in the microemulsion system. This was due to the lower interfacial tension between oleic acid (oil phase) and water in the presence of surfactant in the microemulsion system. This led to the formation of a stable microemulsion system and facilitated a higher loading of curcumin onto starch nanoparticles [40, 41].

The loading efficiency of curcumin onto starch nanoparticles was observed to increase when the concentrations of Tween 80 surfactant increased from 0 to $0.8 \times 10^{-3} \mathrm{M}$. This could be due to an increase in Tween 80 surfactant concentrations that had led to stabilizing the interfacial tension of oleic acid/ethanol microemulsion system and hence increased curcumin loading [42]. However, above
TABLE 2: Effects of surfactant concentration on mean particles diameter of curcumin loaded starch nanoparticles.

\begin{tabular}{lc}
\hline $\begin{array}{l}\text { Surfactant concentration } \\
\left(\times 10^{-3} \mathrm{M}\right)\end{array}$ & Mean particles diameter $(\mathrm{nm})$ \\
\hline 0 & 137 \\
0.4 & 109 \\
0.8 & 83 \\
1.5 & 101 \\
2.3 & 119 \\
\hline
\end{tabular}

$0.8 \times 10^{-3} \mathrm{M}$ of Tween 80 concentration, the loading efficiency started to drop due to the presence of high concentration of surfactant which could be caused by the highly viscous solution system. As a result, larger particles (Table 2) with lower surface area and lower loading efficiency of curcumin were produced $[43,44]$.

The mean particle diameter of curcumin loaded starch nanoparticles prepared in oleic acid/ethanol microemulsion reaction medium with $0.8 \times 10^{-3} \mathrm{M}$ of Tween 80 appeared to be smaller $(83 \mathrm{~nm})$ as compared to sample without surfactant $(137 \mathrm{~nm})($ Table 2). The presence of optimum concentration of surfactant in oleic acid/ethanol microemulsion reduced the surface tension between the starch nanoparticles, which had prevented the coalescence between starch nanoparticles in the microemulsion system and led to the formation of smaller particle sizes. In contrast, curcumin loaded starch nanoparticles of larger particle sizes were formed without the presence of surfactant in the microemulsion system [40,45].

3.2.4. Effect of Oil/Ethanol Ratio. The effect of oil/ethanol volume ratios $(\mathrm{v} / \mathrm{v})$ on the loading efficiency of curcumin onto starch nanoparticles was shown in Figure 7. It was found that as the ratio of oleic acid (oil phase) to ethanol increased, the loading efficiency of curcumin onto starch nanoparticles decreased. This decrease was due to the destabilization of oleic acid/ethanol microemulsion system by the excess oil volume which resulted in the formation of an inhomogeneous and cloudy solution [46]. The optimum ratio of oil to ethanol $(1: 3)$ had resulted in the highest loading efficiency of curcumin onto starch nanoparticles. This could be attributed to the role of ethanol as a cosurfactant which have reduced the interfacial tension of oleic acid/ethanol microemulsion and improved the interactions of Tween 80 surfactant monolayer with water and oil at the interface of oleic acid/ethanol microemulsion system and consequently the formation of a stable microemulsion [47].

Besides, ethanol also served as a cosolvent to enhance the dissolution of curcumin and Tween 80 surfactant in the oleic acid/ethanol microemulsion reaction media and thus improved loading efficiency of curcumin onto starch nanoparticles. Similar observations have been reported by other researchers [48-50], where their studies showed that most of the surfactants alone could not produce stable microemulsion system and the addition of solvent such as alcohol was required to facilitate the formation of a stable microemulsion system. 


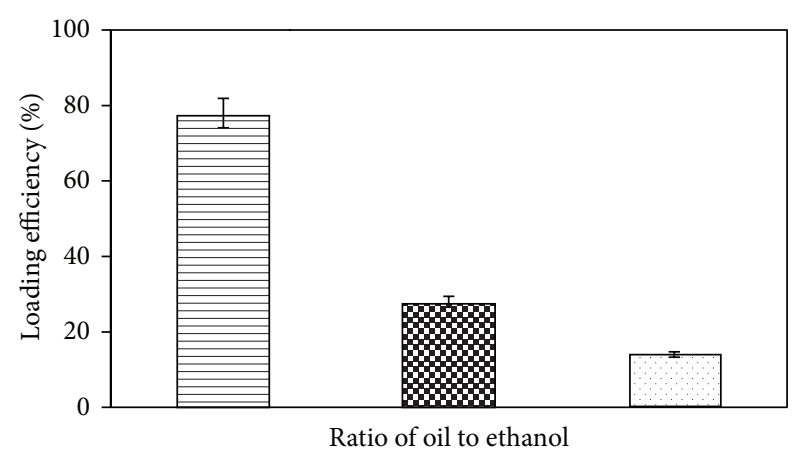

$\boxminus 1: 3$
운 $1: 1$
$\square 3: 1$

FIGURE 7: Effect of oil to ethanol volume (oil : ethanol) on loading efficiency of curcumin onto starch nanoparticles.

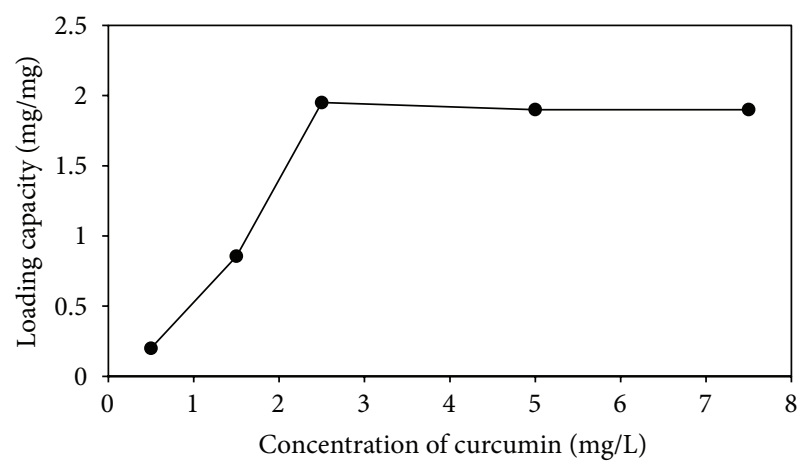

FIGURE 8: Effect of curcumin concentration on loading capacity of curcumin onto starch nanoparticles.

3.2.5. Effect of Curcumin Concentration. Figure 8 demonstrates that the loading capacity of curcumin onto starch nanoparticles increased almost linearly from 0.2 to $2.0 \mathrm{mg} / \mathrm{mg}$ as the concentration of curcumin was increased from 0.5 to $2.5 \mathrm{mg} / \mathrm{L}$. A maximum loading capacity of $2.0 \mathrm{mg} / \mathrm{mg}$ was reached at the curcumin concentration of $2.5 \mathrm{mg} / \mathrm{L}$. This curcumin loading capacity was comparable to other drug carriers such as graphene oxide [51], which indicated that starch nanoparticles were indeed promising drug delivery carriers.

3.3. Curcumin Release Studies. The curcumin loaded starch nanoparticles which were prepared in oleic acid/ethanol microemulsion reaction medium in the presence of $0.8 \times$ $10^{-3} \mathrm{M}$ Tween 80 surfactant were used for release studies. The release studies were performed in buffer solution at $\mathrm{pH} 7.4$ at $37 \pm 0.5^{\circ} \mathrm{C}$ as a function of time with continuous stirring over 10 days $(240 \mathrm{~h})$. Figure 9 shows the swelling behaviour of the starch nanoparticles and the release profile of curcumin from starch nanoparticles.

The release characteristics of curcumin from starch nanoparticles were observed to be dependent on the swelling behaviour of the starch nanoparticles. Starch nanoparticles

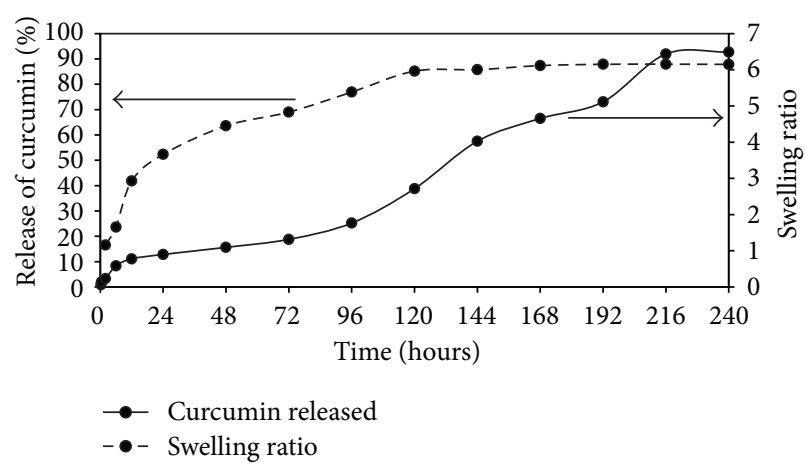

FIGURE 9: Swelling ratio of starch nanoparticles and release profile of curcumin from starch nanoparticles as a function of time.

are very hydrophilic with plenty of $\mathrm{OH}$ group starch nanoparticles swelled due to absorption of the buffer solution and curcumin in the swollen part of starch nanoparticles were diffused out from the starch nanoparticles gradually. Starch nanoparticles were observed to afford a sustained release of curcumin without initial burst release of curcumin being observed. In the first $24 \mathrm{~h}$, starch nanoparticles showed a swelling ratio of 3.66 , and a total of about $13 \%$ curcumin was released gradually out from starch nanoparticles. As the swelling ratio of starch nanoparticles increased to 6.15 , about $93 \%$ of curcumin had been released within a period of $240 \mathrm{~h}$.

\section{Conclusions}

In conclusion, we have successfully loaded curcumin onto starch nanoparticles using a simple technique of nanoprecipitation in microemulsion system. The loading efficiency can be optimized by the judicious choice of reaction medium, types and concentrations of surfactant, oil/ethanol ratios, and curcumin concentration. Loading of curcumin by using $2.5 \times 10^{-5} \mathrm{M}$ of curcumin in $1: 3(\mathrm{v} / \mathrm{v})$ ratio of oleic acid to ethanol microemulsion system with $0.8 \times 10^{-3} \mathrm{M}$ of Tween 80 surfactant and $2 \mathrm{~h}$ loading time was found to give rise to highest loading efficiency of curcumin onto starch nanoparticles. Loading of curcumin onto starch nanoparticles affords a controlled release mechanism with about $93 \%$ of the curcumin being released from starch nanoparticles at the physiological pH 7.4 over 10 days. Overall, this study suggested that starch nanoparticles have the potential to be used as controlled release nanocarriers for curcumin.

\section{Conflict of Interests}

The authors declare that there is no conflict of interests regarding the publication of this paper.

\section{Acknowledgments}

Financial support by Ministry of Higher Education (MOHE) through Fundamental Research Grant Scheme (FRGS), Grant 
no. 01(17)746/2010(32) and MyBrain15 (MyMaster) Programme for graduate scholarship were gratefully acknowledged.

\section{References}

[1] A. B. Kunnumakkara, P. Anand, and B. B. Aggarwal, "Curcumin inhibits proliferation, invasion, angiogenesis and metastasis of different cancers through interaction with multiple cell signaling proteins," Cancer Letters, vol. 269, no. 2, pp. 199-225, 2008.

[2] P. Anand, A. B. Kunnumakkara, R. A. Newman, and B. B. Aggarwal, "Bioavailability of curcumin: problems and promises," Molecular Pharmaceutics, vol. 4, no. 6, pp. 807-818, 2007.

[3] B. B. Aggarwal, A. Kumar, and A. C. Bharti, "Anticancer potential of curcumin: preclinical and clinical studies," Anticancer Research, vol. 23, no. 1 A, pp. 363-398, 2003.

[4] A. Sahu, U. Bora, N. Kasoju, and P. Goswami, "Synthesis of novel biodegradable and self-assembling methoxy poly(ethylene glycol)-palmitate nanocarrier for curcumin delivery to cancer cells," Acta Biomaterialia, vol. 4, no. 6, pp. 1752-1761, 2008.

[5] J. J. Johnson and H. Mukhtar, "Curcumin for chemoprevention of colon cancer," Cancer Letters, vol. 255, no. 2, pp. 170-181, 2007.

[6] A. Shimatsu, H. Kakeya, A. Imaizumi, T. Marimoto, M. Kanai, and S. J. Maeda, "Clinical application of, "curcumin", a multifunctional substance," Anti-Aging Medicine, vol. 9, no. 91, pp. 729-732, 2012.

[7] R. E. Carroll, R. V. Benya, D. K. Turgeon et al., "Phase IIa clinical trial of curcumin for the prevention of colorectal neoplasia," Cancer Prevention Research, vol. 4, no. 3, pp. 354-364, 2011.

[8] S. F. Chin, K. S. Iyer, M. Saunders et al., "Encapsulation and sustained release of curcumin using superparamagnetic silica reservoirs," Chemistry, vol. 15, no. 23, pp. 5661-5665, 2009.

[9] A. Anitha, S. Maya, N. Deepa et al., "Efficient water soluble O-carboxymethyl chitosan nanocarrier for the delivery of curcumin to cancer cells," Carbohydrate Polymers, vol. 83, no. 2, pp. 452-461, 2011.

[10] A. El-Hag Ali and A. AlArifi, "Characterization and in vitro evaluation of starch based hydrogels as carriers for colon specific drug delivery systems," Carbohydrate Polymers, vol. 78, no. 4, pp. 725-730, 2009.

[11] L. Chen, X. Li, L. Li, and S. Guo, "Acetylated starch-based biodegradable materials with potential biomedical applications as drug delivery systems," Current Applied Physics, vol. 7, no. 1, pp. e90-e93, 2007.

[12] A. Szepes, Z. Makai, C. Blümer, K. Mäder, P. Kása Jr., and P. Szabó-Révész, "Characterization and drug delivery behaviour of starch-based hydrogels prepared via isostatic ultrahigh pressure," Carbohydrate Polymers, vol. 72, no. 4, pp. 571-578, 2008.

[13] Q. Wang, N. Zhang, X. Hu, J. Yang, and Y. Du, "Chitosan/starch fibers and their properties for drug controlled release," European Journal of Pharmaceutics and Biopharmaceutics, vol. 66, no. 3, pp. 398-404, 2007.

[14] Q. Wang, X. Hu, Y. Du, and J. F. Kennedy, "Alginate/starch blend fibers and their properties for drug controlled release," Carbohydrate Polymers, vol. 82, no. 3, pp. 842-847, 2010.

[15] M. J. Santander-Ortega, T. Stauner, B. Loretz et al., "Nanoparticles made from novel starch derivatives for transdermal drug delivery," Journal of Controlled Release, vol. 141, no. 1, pp. 85-92, 2010.
[16] S. Y. Xiao, X. M. Liu, C. Y. Tong et al., "Dialdehyde starch nanoparticles as antitumor drug delivery system: an in vitro, in vivo, and immunohistological evaluation," Chinese Science Bulletin, vol. 57, no. 24, pp. 3226-3232, 2012.

[17] S. F. Chin, S. C. Pang, and S. H. Tay, "Size controlled synthesis of starch nanoparticles by a simple nanoprecipitation method," Carbohydrate Polymers, vol. 86, no. 4, pp. 1817-1819, 2011.

[18] M. Boutonnet, J. Kizling, P. Stenius, and G. Maire, "The preparation of monodisperse colloidal metal particles from microemulsions," Colloids and Surfaces, vol. 5, no. 3, pp. 209225, 1982.

[19] E. Caponetti, D. C. Martino, M. Leone, L. Pedone, M. L. Saladino, and V. Vetri, "Microwave-assisted synthesis of anhydrous CdS nanoparticles in a water-oil microemulsion," Journal of Colloid and Interface Science, vol. 304, no. 2, pp. 413-418, 2006.

[20] M. Ethayaraja, K. Dutta, D. Muthukumaran, and R. Bandyopadhyaya, "Nanoparticle formation in water-in-oil microemulsions: experiments, mechanism, and Monte Carlo simulation," Langmuir, vol. 23, no. 6, pp. 3418-3423, 2007.

[21] R. K. Das, N. Kasoju, and U. Bora, "Encapsulation of curcumin in alginate-chitosan-pluronic composite nanoparticles for delivery to cancer cells," Nanomedicine, vol. 6, no. 1, pp. e153e160, 2010.

[22] K. G. H. Desai and H. J. Park, "Preparation and characterization of drug-loaded chitosan-tripolyphosphate microspheres by spray drying," Drug Development Research, vol. 64, no. 2, pp. 114-128, 2005.

[23] R. K. Dutta and S. Sahu, "Development of oxaliplatin encapsulated in magnetic nanocarriers of pectin as a potential targeted drug delivery for cancer therapy," Results in Pharma Sciences, vol. 2, pp. 38-45, 2012.

[24] A. Dev, N. S. Binulal, A. Anitha et al., "Preparation of poly(lactic acid)/chitosan nanoparticles for anti-HIV drug delivery applications," Carbohydrate Polymers, vol. 80, no. 3, pp. 833-838, 2010.

[25] M. E. Mathew, J. C. Mohan, K. Manzoor, S. V. Nair, H. Tamura, and R. Jayakumar, "Folate conjugated carboxymethyl chitosanmanganese doped zinc sulphide nanoparticles for targeted drug delivery and imaging of cancer cells," Carbohydrate Polymers, vol. 80, no. 2, pp. 442-448, 2010.

[26] S. C. Pang, S. F. Chin, S. H. Tay, and F. M. Tchong, "Starchmaleate-polyvinyl alcohol hydrogels with controllable swelling behaviors," Carbohydrate Polymers, vol. 84, no. 1, pp. 424-429, 2011.

[27] G. H. Sagar, M. A. Arunagirinathan, and J. R. Bellare, "Selfassembled surfactant nano-structures important in drug delivery: a review," Indian Journal of Experimental Biology, vol. 45, no. 2, pp. 133-159, 2007.

[28] N. Suwannateep, W. Banlunara, S. P. Wanichwecharungruang, K. Chiablaem, K. Lirdprapamongkol, and J. Svasti, "Mucoadhesive curcumin nanospheres: biological activity, adhesion to stomach mucosa and release of curcumin into the circulation," Journal of Controlled Release, vol. 151, no. 2, pp. 176-182, 2011.

[29] A. Mukerjee and J. K. Vishwanatha, "Formulation, characterization and evaluation of curcumin-loaded PLGA nanospheres for cancer therapy," Anticancer Research, vol. 29, no. 10, pp. 38673875, 2009.

[30] L. Peltonen, P. Koistinen, M. Karjalainen, A. Häkkinen, and J. Hirvonen, "The effect of cosolvents on the formulation of nanoparticles from low-molecular-weight poly(l)lactide," AAPS PharmSciTech, vol. 3, no. 4, pp. E1-E7, 2002. 
[31] H. Yu and Q. Huang, "Enhanced in vitro anti-cancer activity of curcumin encapsulated in hydrophobically modified starch," Food Chemistry, vol. 119, no. 2, pp. 669-674, 2010.

[32] L. M. Huong, H. P. Thu, N. T. B. Thuy et al., "Preparation and antitumor-promoting activity of curcumin encapsulated by $1,3-\beta$-glucan isolated from Vietnam medicinal mushroom hericium erinaceum," Chemistry Letters, vol. 40, no. 8, pp. 846848, 2011.

[33] C. Malcolmson and M. J. Lawrence, "A comparison of the incorporation of model steroids into non-ionic micellar and microemulsion systems," Journal of Pharmacy and Pharmacology, vol. 45, no. 2, pp. 141-143, 1993.

[34] K. Shinoda and B. Lindman, "Organized surfactant systems: microemulsions," Langmuir, vol. 3, no. 2, pp. 135-149, 1987.

[35] C. Tojo, M. Dios, and F. Barroso, "Surfactant effects on microemulsion-based nanoparticle synthesis," Materials, vol. 4, no. 1, pp. 55-72, 2010.

[36] E. Dickinson, "Hydrocolloids at interfaces and the influence on the properties of dispersed systems," Food Hydrocolloids, vol. 17, no. 1, pp. 25-39, 2003.

[37] M. Wulff-Pérez, A. Torcello-Gómez, M. J. Gálvez-Ruíz, and A. Martín-Rodríguez, "Stability of emulsions for parenteral feeding: preparation and characterization of o/w nanoemulsions with natural oils and Pluronic f68 as surfactant," Food Hydrocolloids, vol. 23, no. 4, pp. 1096-1102, 2009.

[38] A. Manosroi, P. Wongtrakul, J. Manosroi et al., "Characterization of vesicles prepared with various non-ionic surfactants mixed with cholesterol," Colloids and Surfaces B, vol. 30, no. 1-2, pp. 129-138, 2003.

[39] T. Schmidts, D. Dobler, C. Nissing, and F. Runkel, "Influence of hydrophilic surfactants on the properties of multiple W/O/W emulsions," Journal of Colloid and Interface Science, vol. 338, no. 1, pp. 184-192, 2009.

[40] C.-Y. Huang, C.-M. Chen, and Y.-D. Lee, "Synthesis of high loading and encapsulation efficient paclitaxel-loaded poly(nbutyl cyanoacrylate) nanoparticles via miniemulsion," International Journal of Pharmaceutics, vol. 338, no. 1-2, pp. 267-275, 2007.

[41] S. K. Jha, S. Dey, and R. Karki, "Microemulsions-potential carrier for improved drug delivery," Asian Journal of Biomedical and Pharmaceutical Sciences, vol. 1, no. 2, pp. 5-9, 2011.

[42] J.-M. Jin, K. Parbhakar, and L. H. Dao, "Model for water-in-oil microemulsions: surfactant effects," Physical Review E, vol. 55, no. 1, pp. 721-726, 1997.

[43] M. Beck-Broichsitter, E. Rytting, T. Lebhardt, X. Wang, and T. Kissel, "Preparation of nanoparticles by solvent displacement for drug delivery: a shift in the "ouzo region" upon drug loading," European Journal of Pharmaceutical Sciences, vol. 41, no. 2, pp. 244-253, 2010.

[44] L. Francis, D. Meng, J. Knowles, T. Keshavarz, A. R. Boccaccini, and I. Roy, "Controlled delivery of gentamicin using poly(3-hydroxybutyrate) microspheres," International Journal of Molecular Sciences, vol. 12, no. 7, pp. 4294-4314, 2011.

[45] L. Pachuau and B. Mazumder, "A study on the effects of different surfactants on Ethylcellulose microspheres," International Journal of PharmTech Research, vol. 1, no. 4, pp. 966-971, 2009.

[46] A. Rukmini, S. Raharjo, P. Hastuti, and S. Supriyadi, "Formulation and stability of water-in-virgin coconut oil microemulsion using ternary food grade nonionic surfactants," International Food Research Journal, vol. 19, no. 1, pp. 259-264, 2012.
[47] R. G. Alany, T. Rades, S. Agatonovic-Kustrin, N. M. Davies, and I. G. Tucker, "Effects of alcohols and diols on the phase behaviour of quaternary systems," International Journal of Pharmaceutics, vol. 196, no. 2, pp. 141-145, 2000.

[48] A. Jada, J. Lang, R. Zana, R. Makhloufi, E. Hirsch, and S. J. Candau, "Ternary water in oil microemulsions made of cationic surfactants, water, and aromatic solvents. 2. Droplet sizes and interactions and exchange of material between droplets," Journal of Physical Chemistry, vol. 94, no. 1, pp. 387-395, 1990.

[49] J. Lang, N. Lalem, and R. Zana, "Quaternary water in oil microemulsions. 1. Effect of alcohol chain length and concentration on droplet size and exchange of material between droplets," Journal of Physical Chemistry, vol. 95, no. 23, pp. 9533-9541, 1991.

[50] D. S. Mathew and R.-S. Juang, "Role of alcohols in the formation of inverse microemulsions and back extraction of proteins/enzymes in a reverse micellar system," Separation and Purification Technology, vol. 53, no. 3, pp. 199-215, 2007.

[51] X. Yang, X. Zhang, Z. Liu, Y. Ma, Y. Huang, and Y. Chen, "High-efficiency loading and controlled release of doxorubicin hydrochloride on graphene oxide," Journal of Physical Chemistry C, vol. 112, no. 45, pp. 17554-17558, 2008. 

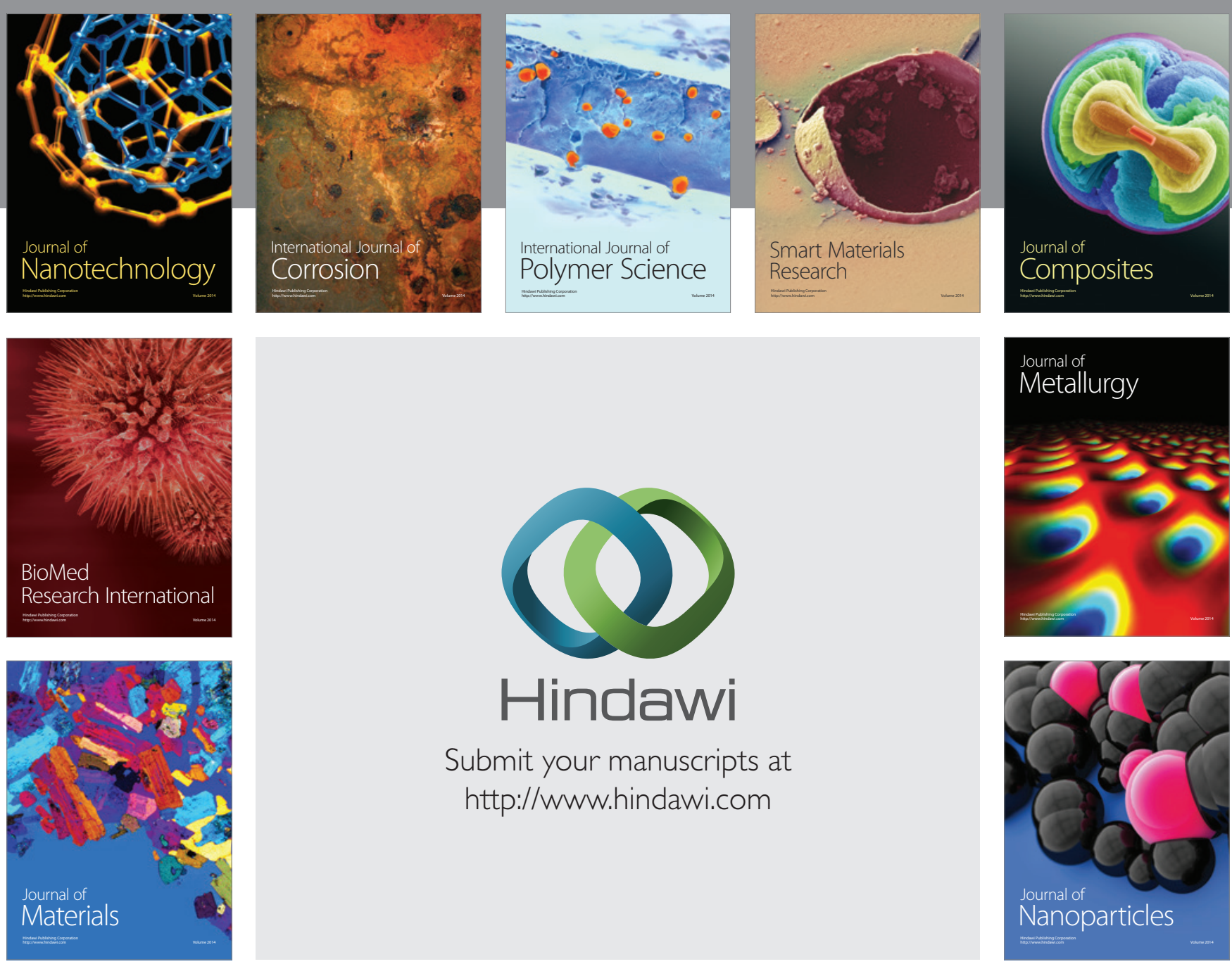

Submit your manuscripts at http://www.hindawi.com
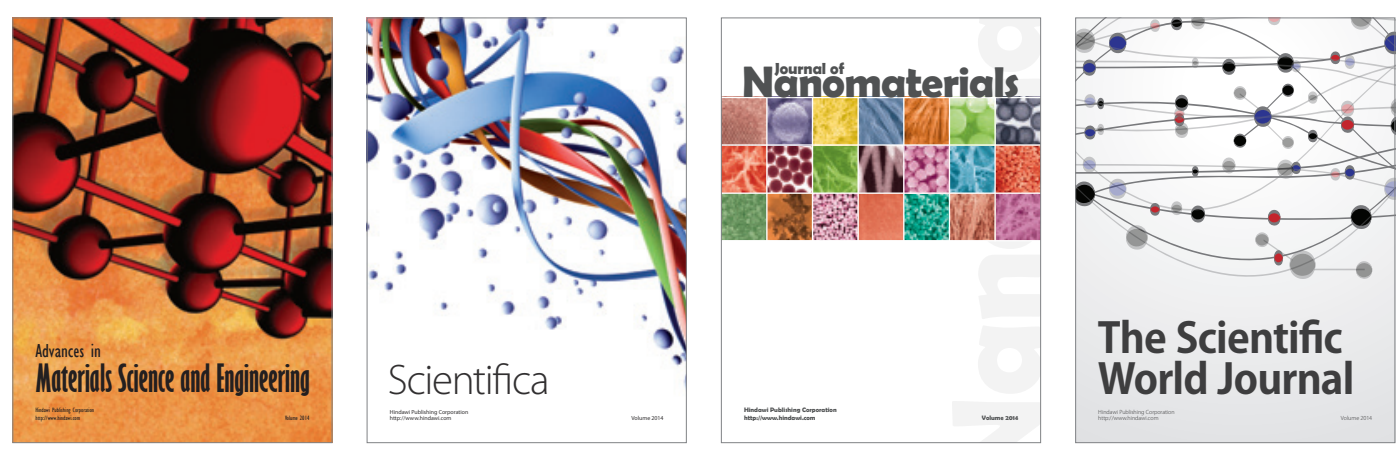

\section{The Scientific World Journal}
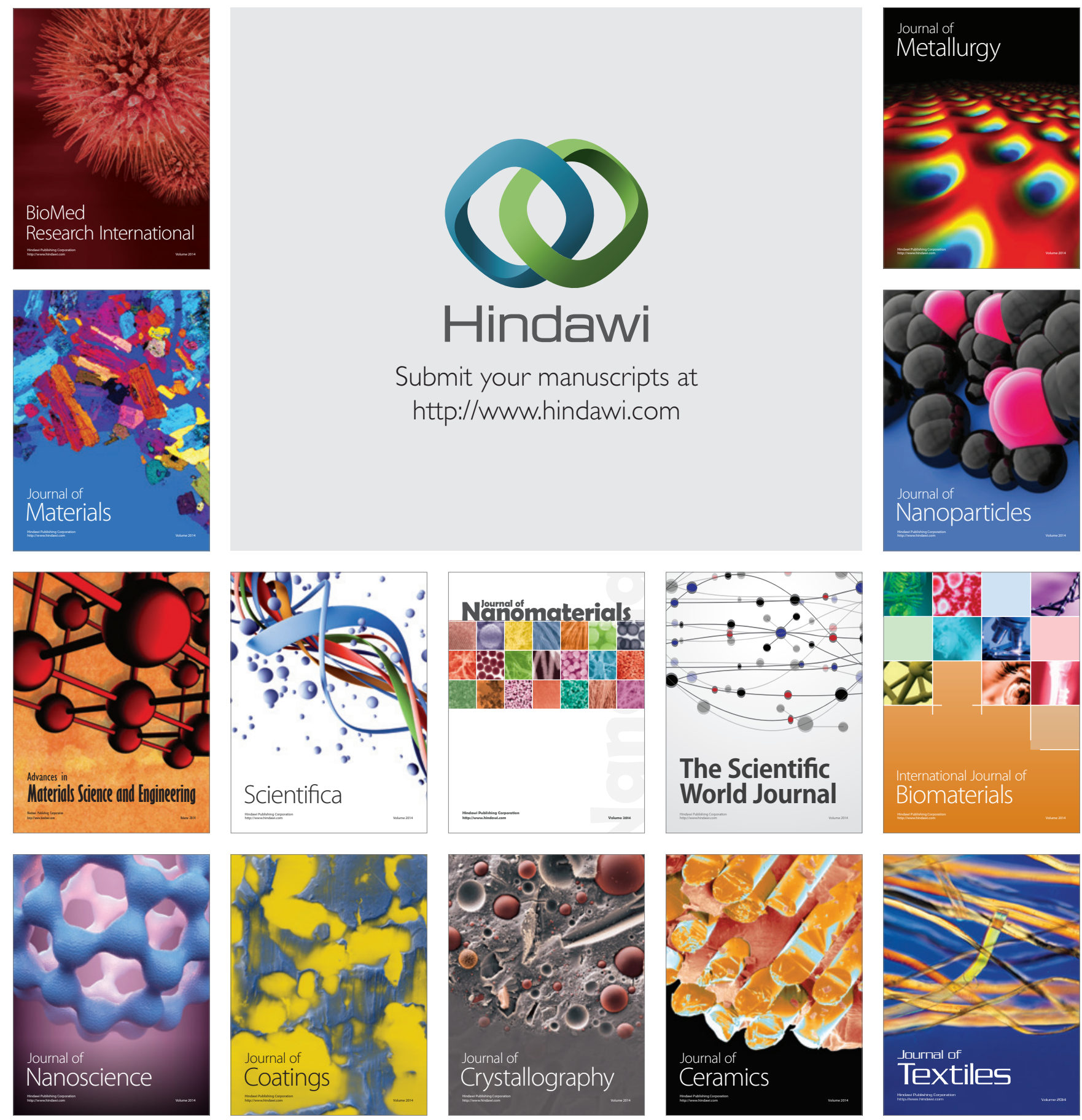